http://dx.doi.org/10.32929/2446-8355.2019v28n3p367-375

\title{
OCORRÊNCIA DE PLANTAS ESPONTÂNEAS DA VEGETAÇÃO NATURAL NA SAVANA AMAZÔNICA, BRASIL
}

\section{Djair Alves de Melo ${ }^{1 *}$, Tocio Sediyama ${ }^{2}$, José Maria Arcanjo Alves ${ }^{3}$, José de Anchieta Alves de Albuquerque ${ }^{3}$, Antônio Alberto da Silva², Sandra Cátia Pereira Uchôa ${ }^{3}$}

1 Docente, Departamento de Agroecologia, IFPB, Picuí-PB. *E-mail do autor correspondente: djairalves@hotmail.com

${ }^{2}$ Docente, Departamento de Fitotecnia, Universidade Federal de Viçosa, Viçosa-MG.

${ }^{3}$ Docente, Departamento de Fitotecnia, Universidade Federal de Roraima, Boa Vista-RR.

Recebido: 18/06/19; Aceito: 22/10/2019

RESUMO: Objetivou-se com este trabalho avaliar a ocorrência de plantas espontâneas em área de vegetação natural na savana Amazônica não incorporada ao sistema produtivo. As plantas espontâneas foram identificadas quanto à classe botânica, família e espécie, sendo quantificadas por meio do somatório das amostras coletadas ao acaso na área experimental. Para os parâmetros fitossociológicos, foram considerados: frequência, frequência relativa, densidade, densidade relativa, abundância, abundância relativa, índice de valor de importância (IVI\%) e importância relativa (IR\%). As plantas espontâneas foram distribuídas em nove famílias, 14 gêneros e 15 espécies. A família com maior número de espécies foi Fabaceae e a família com maior número de indivíduos por espécie foi Poaceae. As espécies Axonopus aureus e Trachypogon plumus apresentam os maiores parâmetros fitossociológicos. Recomenda-se outras pesquisas nas áreas da vegetação natural na savana Amazônica não incorporada ao sistema produtivo em virtude dos poucos trabalhos realizados.

Palavras-chave: Fitossociologia. Espécies nativas. Axonopus aureus. Trachypogon plumus.

\section{OCCURRENCE OF WEEDS OF NATURAL VEGETATION IN SAVANA AMAZÔNICA, BRAZIL}

\begin{abstract}
The objective of this work was to evaluate the occurrence of spontaneous plants in an area of natural vegetation in the Amazonian savannah not incorporated into the productive system. The spontaneous plants were identified as botanical class, family and species, being quantified by the sum of the samples collected at random in the experimental area. For the phytosociological parameters, frequency, relative frequency, density, relative density, abundance, relative abundance, importance value index (IVI\%) and relative importance (IR\%) were considered. Spontaneous plants were distributed in nine families, 14 genera and 15 species. The family with the highest number of species was Fabaceae and the family with the highest number of individuals per species was Poaceae. The species Axonopus aureus and Trachypogon plumus present the highest phytosociological parameters. Other research is recommended in the areas of natural vegetation in the Amazonian savannah not incorporated to the productive system due to the few works carried out.
\end{abstract}


Keywords: Phytosociology. Native species. Axonopus aureus. Trachypogon plumus.

\section{INTRODUÇÃO}

O Estado de Roraima possui aproximadamente 1,5 milhões de hectares e geograficamente localizado no extremo norte do Brasil na sua região fronteiriça com os países da Guiana Inglesa e Venezuela. Dentre os ecossistemas encontrados no Estado se destaca as Savanas Roraimenses que possuir a maior área de savana da Amazônia brasileira, formando o complexo paisagístico "Rio Branco-Rupununi", que está em uma faixa de terra da Guiana Inglesa até a Venezuela (BARBOSA et al., 2007).

A savana de Roraima apresenta grande heterogeneidade botânica de plantas espontâneas e de acordo com o Ministério do Meio Ambiente - MMA (2017) a savana brasileira é reconhecida mundialmente como a mais rica, tendo aproximadamente 11.800 espécies de plantas nativas catalogadas. Destas mais de 300 espécies têm uso medicinal e mais 450 podem ser usadas na recuperação de solos degradados, sendo o bioma brasileiro que mais sofreu alterações com a ocupação humana nos últimos 30 anos.

A disponibilidade de nutrientes, a alta concentração de alumínio trocável, assim como o baixo $\mathrm{pH}$ do solo podem atuar como filtros na seleção de espécies, já que elas respondem de forma individual às variáveis ambientais nas quais estão inseridas. Desse modo, essas espécies podem indicar indiretamente a qualidade do solo e auxiliar a compreensão do gradiente estrutural entre as fisionomias, uma vez que espécies indicadoras são aquelas que respondem às mudanças do meio através do declínio, desaparecimento ou abundância no crescimento e no aumento da capacidade de reprodução (LACHER, 2000).

Dentre os inúmeros grupos de plantas no meio ambiente, se destacam as plantas espontâneas. Estas muitas vezes são referenciadas como "daninhas ou invasoras", ocorrendo espontaneamente em áreas livres de cultivos ou em áreas onde ocorre exploração agrícola. As espécies invasoras podem ser denominadas exóticas, as quais apresentam alta capacidade de crescimento, proliferação e dispersão, capazes de modificar a composição, estrutura ou função do ecossistema (CRONK, 1995). Uma planta espontânea, ou nativa, pode ser considerada daninha, devido a algum desequilíbrio ecológico, passando a crescer de forma descontrolada, comportando-se como invasora (MATOS; PIVELLO, 2009).

O levantamento florístico, que consiste em identificar e catalogar espécies de plantas de uma determinada área, com a finalidade de se obter um arquivo de nomes populares e científicos das espécies encontradas durante a pesquisa in loco, proporcionando a elaboração de recurso visual, informativo, didático e pedagógico; de valia incalculável, com suporte para conhecer, preservar e conservar a biodiversidade florística de cada região (SILVA et al., 2010).

Alguns trabalhos de pesquisa realizados em área de savana do estado de Roraima com o intuito de levantar o banco florístico da mesma, no entanto, os trabalhos realizados para levantamento dos parâmetros fitossociológicos se restringem às áreas já cultivadas (CRUZ et al., 2009; ALBUQUERQUE et al., 2013; ALBUQUERQUE et al., 2014; EVANGELISTA et al., 2015; ALBUQUERQUE et al., 2017a; ALBUQUERQUE et al., 2017b; GONZAGA et 
al., 2018; CRUZ et al., 2018). Porém existem poucos trabalhos que contemplam o levantamento fitossociológico das espécies vegetais da savana de Roraima ainda não incorporada ao sistema produtivo (MIRANDA; ABSY, 1997; MIRANDA; ABSY, 2000; FLORES; RODRIGUES, 2010).

Diante do exposto, objetivou-se com este trabalho avaliar a ocorrência de plantas espontâneas em área de vegetação natural na savana Amazônica não incorporada ao sistema produtivo.

\section{MATERIAL E MÉTODOS}

O trabalho foi realizado em solo classificado como Latossolo Amarelo distrócoeso em superfície de aplainamento Quartenária, denominada Pediplano Rio Branco, com cotas altimétricas entre 60 a $160 \mathrm{~m}$ (BENEDETTI et al., 2011), não incorporado ao sistema produtivo e localizado na área experimental do Centro de Ciências Agrárias da Universidade Federal de Roraima - CCA/UFRR, no Campus Cauamé, município de Boa Vista em Roraima - Brasil (Latitude de $2^{\circ} 52^{\prime} 15,49^{\prime}$ N, Longitude 6042’39,89’W e Altitude de $85 \mathrm{~m}$ ).

O clima da região é classificado conforme Köppen com Aw, definido como tropical úmido. A precipitação média anual é de $1.678 \mathrm{~mm}$, umidade relativa do ar de $70 \%$ e a temperatura entre 20 a $38^{\circ} \mathrm{C}$, sendo a média anual de $27,4{ }^{\circ} \mathrm{C}$ (ARAÚJO et al., 2001).

O levantamento fitossociológico das plantas espontâneas foi realizado em uma área de vegetação natural através de 40 pontos de amostragem em área de $1.157 \mathrm{~m}^{2}$ com um quadrado de ferro com $0,25 \mathrm{~m}^{2}(0,5 \times 0,5 \mathrm{~m})$, por meio da metodologia do Quadrado Inventário proposto por Braun-Blanquet (1979).

As plantas espontâneas coletadas foram cortadas ao nível do solo, separadas e levadas ao Laboratório de Grandes Culturas do CCA/UFRR, onde foram identificadas por família e espécie (APG, 2009), fazendo-se a contagem. Com base na identificação e contagem das espécies foi realizado o cálculo das seguintes variáveis fitossociológicas: frequência, frequência relativa, densidade, densidade relativa, abundância, abundância relativa, índice de valor de importância e índice de importância relativa (BRANDÃO et al., 1998).

Para determinação dos parâmetros fitossociológicos foram considerados: frequência $(\mathrm{F})$ = número de parcelas que contém a espécie/número total das parcelas utilizadas; densidade (D) = número total de indivíduos por espécie/área total coletada; dominância $(\mathrm{DO})$ = número total de indivíduos por espécie/número total de parcelas que contém a espécie; frequência relativa $(\mathrm{Fr}, \%)=$ frequência da espécie $\mathrm{x}$ 100/frequência total de todas as espécies; densidade relativa $(\mathrm{Dr}, \%)=$ densidade da espécie $\mathrm{x}$ 100/densidade total de todas as espécies; Dominância relativa $(\mathrm{DR}, \%)=$ dominância da espécie $\mathrm{x}$ 100/dominância total de todas as espécies, índice de valor de importância (IVI, \%) = Fr + Dr + Ar (MUELLER-DUMBOIS; ELLENBERG, 1974) e importância relativa (IR) $=100 \mathrm{x}$ índice de valor de importância da espécie $\div$ índice de valor de importância total de todas as espécies (MOURA FILHO et al., 2015). 


\section{RESULTADOS E DISCUSSÃO}

Foram identificadas 15 espécies distribuídas em nove famílias. Com maiores números de espécies foram Fabaceae, Poaceae e Cyperaceae, sendo a Fabaceae que apresentou o maior número de espécies. Relacionado à classe botânica, predominou a Dicotiledoneae (Tabela 1).

Tabela 1. Família, nome científico, nome comum e classe botânica da vegetação espontânea em área de savana não incorporada ao sistema produtivo. Boa Vista, Roraima. Family, scientific name, common name and botanical class of weed in savanna area not incorporated into the productive system. Boa Vista, Roraima.

\begin{tabular}{cccc}
\hline Família & Nome científico & Nome comum & Classe \\
\hline \multirow{2}{*}{ Poaceae } & Trachypogon plumosus & - & Monocotiledoneae \\
& Axonopus aureus & - & Monocotiledoneae \\
\hline \multirow{2}{*}{ Cyperaceae } & Bulbostylis conifera & - & Monocotiledoneae \\
& Bulbostylis warei & - & Monocotiledoneae \\
\hline \multirow{2}{*}{ Fabaceae } & Galactia jussiaeana & - & Dicotiledoneae \\
& Chamaecrista diphylla & - & Dicotiledoneae \\
& Clitoria guianensis & Mineirão & Dicotiledoneae \\
& Lippia microphyla & Alecrim-da-chapada; & Dicotiledoneae \\
Verbenaceae & Alecrim-do-tabuleiro & Dicotiledoneae \\
\hline Convolvulaceae & Evolvulus sericeus & - & Dicotiledoneae \\
\hline Menispermaceae & Cissampelos ovalifolia & Orelha de onça & Dicotiledoneae \\
\hline Dilleniaceae & Davilla aspera & Cipó-de-fogo & Dicotiledoneae \\
\hline Myrtaceae & Eugenia puniccifolia & Goiabarana & Dicotiledoneae \\
\hline Rubiaceae & Palicourea rigida & Chapeu-de-couro & Dicotiledoneae \\
\hline - & Não identificada & - & Dicotiledoneae \\
\hline
\end{tabular}

As espécies Axonopus aureus, Trachypogon plumosus, Bulbostylis conifera e Bulbostylis warei foram as mais representativas da área, com 117, 98, 49 e 34 indivíduos $/ \mathrm{m}^{2}$ (Tabela 2). A espécie Trachypogon plumosus foi a única encontrada em todas amostras coletadas, com frequência igual a um e a Axonopus aureus foi encontradas em quase todas, com a frequência igual a 0,95. Para a densidade e dominância destacaram as Axonopus aureus e Trachypogon plumosus (Tabela 2).

Ambas as espécies também apresentam maiores frequência relativa, dominância relativa, índice de valor de importância relativa (Tabela 3). 
Tabela 2. Número de indivíduos (NI), frequência (F), densidade (D) e dominância (DO) e de plantas espontâneas em área de savana não incorporada ao sistema produtivo. Boa Vista, Roraima. Number of individuals (NI), frequency $(F)$, density $(D)$ and dominance $(O D)$ and weed in savanna area not incorporated into the productive system. Boa Vista, Roraima.

\begin{tabular}{ccccc}
\hline Espécies & $\mathrm{NI}\left(\mathrm{m}^{2}\right)$ & $\mathrm{F}$ & $\mathrm{D}$ & $\mathrm{DO}$ \\
\hline Axonopus aureus & 117 & 0,95 & 23,40 & 5,85 \\
\hline Trachypogon plumosus & 98 & 1,00 & 19,60 & 4,90 \\
\hline Bulbostylis conifera & 49 & 0,65 & 9,80 & 0,40 \\
\hline Bulbostylis warei & 34 & 0,65 & 6,80 & 1,70 \\
\hline NID & 33 & 0,50 & 6,60 & 1,65 \\
\hline Lippia microphyla & 32 & 0,55 & 6,40 & 1,60 \\
\hline Evolvulus Sericeus & 20 & 0,25 & 4,00 & 1,00 \\
\hline Galactea jussiaeana & 15 & 0,05 & 3,00 & 0,75 \\
\hline Chamaecrista diphylla & 6 & 0,25 & 1,20 & 0,30 \\
\hline Cissampelos ovalifolia & 5 & 0,10 & 1,00 & 0,25 \\
\hline Davilla aspera & 4 & 0,05 & 0,80 & 0,20 \\
\hline Eugenia puniccifolia & 1 & 0,05 & 0,20 & 0,05 \\
\hline Clitoria guianense & 1 & 0,05 & 0,20 & 0,05 \\
\hline Stylosanthes guianensis & 1 & 0,05 & 0,20 & 0,05 \\
\hline Palicourea rigida & 1 & 0,05 & 0,20 & 0,05 \\
\hline TOTAL & 417 & 5,20 & 83,40 & 18,80 \\
\hline
\end{tabular}

A maior presença destas três famílias (Fabaceae, Poaceae e Cyperaceae) na área de estudo vem corroborar os resultados obtidos por Miranda e Absy (2000), Barbosa et al., (2007) e Magnusson et al. (2008) em áreas da savana amazônica. No Brasil, a Fabaceae é a família mais bem representada com 2.807 espécies agrupadas em 222 gêneros abundantes em quase todos os biomas e ecossistemas do país (BRAZIL FLORA GROUP - BFG, 2015).

De acordo com Miranda e Absy (1997; 2000), as Poaceae (Gramineae) são frequentemente encontradas em levantamentos de plantas espontâneas nas savanas do norte da América do Sul, enquanto que as Cyperaceae são muito mais frequentes no estado de Roraima do que nos cerrados do Brasil Central.

A espécie da Poaceae apresenta grande quantidade de diásporos, o que facilita a sua disseminação e consequentemente dificulta o seu controle (LORENZI, 2008). De acordo com Costa et al. (2014) estas espécies compõem aproximadamente $80 \%$ da composição botânica em pastagens nativas na savana do estado de Roraima. Ambas espécies apresentam ciclo de vida perene, hábito de crescimento cespitoso, folhas pilosas com 0,60 m de altura (COSTA et al., 2011). 
Tabela 3. Frequência relativa (Fr, \%), dominância relativa (DR), \%), índice de valor de importância (IVI, \%) e importância relativa (IR) de plantas espontâneas em área de savana não incorporada ao sistema produtivo. Boa Vista, Roraima. Relative dominance $(R P, \%)$, relative dominance (DR),\%), importance value index (IVI,\%) and relative importance (IR) of weed in savanna area not incorporated into the productive system. Boa Vista, Roraima.

\begin{tabular}{ccccc}
\hline Espécies & Fr $(\%)$ & DR $(\%)$ & IVI $(\%)$ & IR (\%) \\
\hline Axonopus aureus & 18,26 & 31,11 & 77,44 & 25,81 \\
\hline Trachypogon plumosus & 19,23 & 26,06 & 68,79 & 22,93 \\
\hline Bulbostylis conifera & 12,50 & 2,12 & 26,37 & 8,79 \\
\hline Bulbostylis warei & 12,50 & 9,04 & 29,69 & 9,89 \\
\hline NID & 9,61 & 8,77 & 26,30 & 8,76 \\
\hline Lippia microphyla & 10,57 & 8,51 & 26,76 & 8,92 \\
\hline Evolvulus Sericeus & 4,80 & 5,31 & 14,92 & 4,97 \\
\hline Galactea jussiaeana & 0,96 & 3,98 & 8,54 & 2,84 \\
\hline Chamaecrista diphylla & 4,80 & 1,59 & 7,84 & 2,61 \\
\hline Cissampelos ovalifolia & 1,92 & 1,32 & 4,45 & 1,48 \\
\hline Davilla aspera & 0,96 & 1,0638 & 2,98 & 0,99 \\
\hline Eugenia puniccifolia & 0,96 & 0,266 & 1,46 & 0,48 \\
\hline Clitoria guianense & 0,96 & 0,266 & 1,46 & 0,48 \\
\hline Stylosanthes guianensis & 0,96 & 0,266 & 1,46 & 0,48 \\
\hline Palicourea rigida & 0,965 & 0,266 & 1,46 & 0,48 \\
\hline TOTAL & 100 & 100 & 300 & 100 \\
\hline
\end{tabular}

Na composição botânica das pastagens nativas da savana de Roraima predomina as espécies da família Poaceae: Trachypogon plumosus e Axonopus aureus, devido ao grande banco de sementes dessas espécies, representando cerca de 70 e 30\%, respectivamente (COSTA et al., 2008; COSTA et al., 2011; COSTA et al., 2013). Magnusson et al. (2008) avaliaram a composição florística e cobertura vegetal de uma área da savana amazônica na região de Alter do Chão, Santarém-PA, observaram que a espécie Trachypogon plumosus encontrava-se presente em quase todas as amostras coletadas, ou seja, apresentando uma elevada frequência de 0,8 .

A importância relativa infere quais espécies são mais importantes em termos de infestação (PITELLI, 2000), levando em consideração a distribuição das espécies, o número de indivíduos e a concentração na área. Os índices fitossociológicos são fundamentais para analisar o impacto que os sistemas de manejo e as práticas agrícolas exercem sobre a dinâmica de crescimento e ocupação de comunidades infestantes em agroecossistemas (GOMES et al., 2010). 


\section{CONCLUSÃO}

A classe botânica predominante é a Dicotiledoneae com $73 \%$ das espécies encontradas.

A família com maior número de espécies é a Fabaceae, entretanto com maior número de indivíduos por espécie é Poaceae.

As espécies Axonopus aureus e Trachypogon plumus apresentam os maiores parâmetros fitossociológicos.

Recomenda-se outras pesquisas nas áreas da vegetação natural na savana Amazônica não incorporada ao sistema produtivo em virtude dos poucos trabalhos realizados.

\section{REFERÊNCIAS BIBLIOGRÁFICAS}

ALBUQUERQUE, J. A. A.; MELO, V. R.; SOARES, M. B. B.; FINOTO, E. L.; SIQUEIRA, R. H. S.; MARTINS, S. A. Fitossociologia e características morfológicas de plantas daninhas após cultivo de milho em plantio convencional no cerrado de Roraima. Revista Agro@mbiente, Boa Vista, v. 7, n. 3, p.313-321, 2013.

AlBUQUERQUE, J. A. A.; SANTOS, T. S. D.; CASTRO, T. S.; EVANGELISTA, M. O.; ALVES, J. M. A.; MENEZES, P. H. S. D. Estudo florístico de plantas daninhas em cultivos de melancia na Savana de Roraima, Brasil. Scientia Agropecuaria, Trujillo, v. 8, n. 2, p.9198, 2017a. Disponível em: DOI: 10.17268/sci.agropecu.2017.02.01. Acesso em: 01 mar 2019.

AlBuQuerque, J. A. A., SANTOS, T. S., CASTRO, T. S., MELO, V. F., \& ROCHA, P. R. R. Weed incidence after soybean harvest in no-till and conventional tillage croprotation systems in Roraima's cerrado. Planta Daninha, Viçosa, v. 35, n. e017162796, p.1-12, 2017 b. DOI: org/10.1590/s0100-83582017350100034. Acesso em: 01 mar 2019.

ANGIOSPERM PHYLOGENY GROUP - APG. An update of the Angiosperm Phylogeny Group classification for the orders and families of flowering plants. APG III. Botanical Journal of the Linnean Society, London, v. 161, n. 2, p.105-121, 2009. Disponível em: DOI: org/10.1111/j.1095-8339.2009.00996.x. Acesso em: 01 mar 2019.

ARAÚJO, W. F.; ANDRADE JÚNIOR, A. S.; MEDEIROS, R. D.; SAMPAIO, R. A. Precipitação pluviométrica mensal provável em Boa Vista, Estado de Roraima, Brasil. Revista Brasileira de Engenharia Agrícola e Ambiental, Campina Grande, v. 5, n. 3, p.563-567, 2001. CDOI: http://dx.doi.org/10.1590/S1415-43662001000300032 Acesso em: 01 mar 2019.

BARBOSA, R. I.; CAMPOS, C.; PINTO, F.; FEARNSIDE, P. M. The "Lavrados" of Roraima: Biodiversity and Conservation of Brazil's Amazonian Savannas. Functional Ecosystems and Communities, v. 1, n. 1, p. 29-41, 2007.

BENEDETTI, U. G.; VALE JÚNIOR, J. F.; SHAEFER, C. E. G. R.; MELO, V. F.; UCHÔA, S. C. Gênese, química e mineralogia de solos derivados de sedimentos pliopleistocênicos e de rochas vulcânicas básicas em Roraima, norte da amazônico. Revista Brasileira de Ciência do Solo, Viçosa, v. 35, n. 35, p.299-212, 2011. 
BRAZIL FLORA GROUP - BFG. Growing knowledge: an overview of Seed Plant diversity in Brazil. Rodriguésia, Rio de Janeiro, v. 66, n. 4, p.1085-1113, 2015.

BRANDÃO, M.; BRANDÃO, H.; LACA-BUENDIA, J. P. A mata ciliar do rio Sapucaí, município de Santa Rita do Sapucaí-MG: fitossociologia. Daphne, Belo Horizonte, v. 8, n. 4, p.36-38, 1998.

BRAUN-BLANQUET, J. Fitossociologia: bases para el estudio de las comunidades vegetales. Madrid: H. Blume, 1979. 820 p.

COSTA, N. L.; GIANLUPPI, V.; MORAES, A. D. Avaliação da rebrota natural de pastagens de Trachypogon plumosus nos cerrados de Roraima. Scientia Agraria Paranaensis, Londrina, v. 13, n. 1, p.57-54, 2014.

COSta, N. L., PAUlino, V. T.; MAGAlhãeS, J. A. Morfogênese de gramíneas forrageiras na Amazônia Ocidental. Pubvet, Londrina, v. 2, n. 29, p.1-12, 2008. Disponível em: DOI: http://dx.10.5216/cab.v13i1.12803. Acesso em: 01 mar 2019.

COSTA, N. L.; MORAES, A.; OLIVEIRA, R. A.; GIANLUPPI, V.; BENDAHAN, A. B.; MAGALHÃES, J. A. Rendimento potencial de pastagens de Trachypogon plumosus nos cerrados de Roraima. Revista Agro@mbiente, Boa Vista, v. 5, n. 3, p.200-206, 2011.

COStA, N. L., MORAES, A., CARVAlhO, P. C. F., MONTEIRO, A. L. G., MOTTA, A. C. V.; OLIVEIRA, R. A. Composição e extração de nutrientes por Trachypogon plumosus sob adubação e idades de rebrota. Archivos de Zootecnia, Córdoba, v. 62, n. 238, p.227-238, 2013.

CRONK, Q. C. B; FULLER, J. L. Plant invaders. London: Chapman \& Hall, 1995. 256 p.

CRUZ, A. B. SOUZA.; ROCHA, P. R. R.; AlBUQUERQUE, J. A. A.; ALVES, J. M. A.; CRUZ, D. L. S.; FINOTO, E. L.; SANTOS, G. X. L. Seletividade de herbicidas aplicados em pré e pós-emergência na cultura do feijão-caupi na savana amazônica. NATIVA, Sinop, v. 6, n. 6, p.625-630, 2018. Disponível em: DOI: http://dx.org/10.31413/nativa.v6i6.5732. Acesso em: 01 mar 2019.

CRUZ, D. L. S.; RODRIGUES, G. S.; DIAS, F. O.; ALVES, J. M. A.; ALBUQUERQUE, J. A. A. Levantamento de plantas daninhas em área rotacionada com as culturas da soja, milho e arroz irrigado no cerrado de Roraima. Revista Agro@mbiente, Boa Vista, v. 3, n. 1, p.58-63, 2009.

EVANGElista, M. O.; ALBUQUERQUE, J. A. A.; SANTOS, C. S. V.; ALVES, J. M. A.; SANTOS, T. S.; CASTRO, T. S.; ALCANTARA NETO, F. Fitossociologia de plantas espontâneas em produção orgânica de hortaliças no estado de Roraima. Revista Sodebras, Brasília, v. 10, n. 117, p.259-266, 2015.

FLORES, A. S.; RODRIGUES, R. S. Diversidade de Leguminosae em uma área de savana do estado de Roraima, Brasil. Acta Amazônica, Manaus, v.24, n. 1, p.175-183, 2010.

GOMES, G. L. G. C.; IBRAHIM, F. N.; MACEDO, G. L.; NOBREGA, L. P.; ALVES, E. Cadastramento fitossociológico de plantas daninhas na bananicultura. Planta Daninha, Viçosa, v. 28, n. 1, p.61-68, 2010. 
GONZAGA, G. S.; SOUZA CRUZ, A. B.; ALBUQUERQUE, J. A. A.; RIBEIRO ROCHA, P. R.; ALVES, J. M. A.; SOUZA, L. T.; SOARES, M. B. B.; SOUZA CRUZ, D. L. Phytosociology of weed communitiy in culture of cowpea [vigna unguiculata L. Walp] and controlling possibilities with pre-emergent herbicides. Applied Ecology and Environmental Research, Budapest, v. 16, n. 5, p.5311-5322, 2018. Disponível em: DOI: http://dx.doi.org/10.15666/aeer/1605_53115322. Acesso em: 01 mar 2019.

LORENZI, H. Plantas daninhas do Brasil: terrestres, aquáticas, parasitas e tóxicas. 4. ed. Nova Odessa: Plantarum, 2008. 640 p.

MAGNUSSON, W. E.; LIMA, A. P.; ALBERNAZ, A. L. K. M.; SANAIOTTI, T. M.; GUILLAUMET, J. L. Composição florística e cobertura vegetal de uma savana amazônica. Revista Brasileira de Botânica, São Paulo, v. 31, n. 1, p.165-177, 2008.

MATOS, D. M. S.; PIVELLO, V. R. O impacto das plantas invasoras nos recursos naturais de ambientes terrestres: alguns casos brasileiros. Ciência e Cultura, Campinas, v. 61, n. 1, p.2730, 2009.

MIRANDA, I. S.; ABSY, M. L. Fisionomia das Savanas de Roraima, Brasil. Acta Amazônica, Manaus, v. 30, n. 3, p.423-440, 2000.

MIRANDA, I. S.; ABSY, M. L. A flora fanerogâmica das savanas de Roraima. In: BARBOSA, R. I.; FERREIRA, E. J. G.; CASTELLÕN, E. G. (Eds.). Homem, ambiente e ecologia no estado de Roraima. Manaus, INPA, 1997. cap. 2, p. 445-462.

MINISTÉRIO DO MEIO AMBIENTE - MMA. O bioma cerrado. 2017. Disponível em: http://www.mma.gov.br/biomas/cerrado/importanciavegetacaonativa2017. Acesso em: 01 mar 2019.

MOURA FILHO, E. R.; MACEDO, L. P. M.; SILVA, A. R. S. Levantamento fitossociológico de plantas daninhas em cultivo de banana irrigada. Holos, Natal, v. 2, n 31. p.92-97, 2015. Disponível em: DOI: 10,15628/holos.2015.1006. Acesso em: 01 mar 2019.

MUELLER-DOMBOIS, D.; ELLENBERG, H. Aims and methods of vegetation ecology. New York: John Wiley e Sons, 1974. 547 p.

PITELLI, R. A. Estudos fitossociológicos em comunidades infestantes de agroecossistemas. Journal of Coservation Science, v. 1, n. 2, p.1-7, 2000.

SILVA, M. A.; BARBOSA, J. S.; ALBUQUERQUE, H. N. Levantamento das plantas espontâneas e suas potencialidades fitoterapêuticas: um estudo no complexo Aluízio Campos - Campina Grande - PB. Revista Brasileira de Informações Científicas, Campina Grande, v. 1, n. 1, p.52- 66, 2010. 